\title{
EDIFICIO DE OFICINAS EN EL PASO ELEVADO DE LAS CALLES EDUARDO DATO-JUAN BRAVO. MADRID/ESPAÑA
}

\author{
(OFFICES BUILDING NEAR BRIDGE JOINING EDUARDO DATO-JUAN BRAVO \\ STREETS IN MADRID/SPAIN)
}

\section{José Ramón Azpiazu Ordóñez \\ Arquitecto}

$(131-146)$

\section{RESUMEN}

En este edificio se ha procurado armonizar el conjunto, pasando de una fachada en muro-cortina con entramado muy pronunciado y tupido, a un muro-cortina completamente liso, mediante una fachada en muro-cortina con un entramado estructural de dimensiones intermedias.

\section{SUMMARY}

This building has been managed so as to get a harmony in the whole of its façades beginning from a curtain-wall with marked and thick framework, to a curtain-wall completely flat by means of a curtain-wall façade with a medium size structural frame.
Entre los edificios del Banco de París y del Banco Peninsular se ha construido este edificio de oficinas de 10 plantas y cuatro sótanos destinados a garaje.

Se ha procurado armonizar el conjunto pasando, de

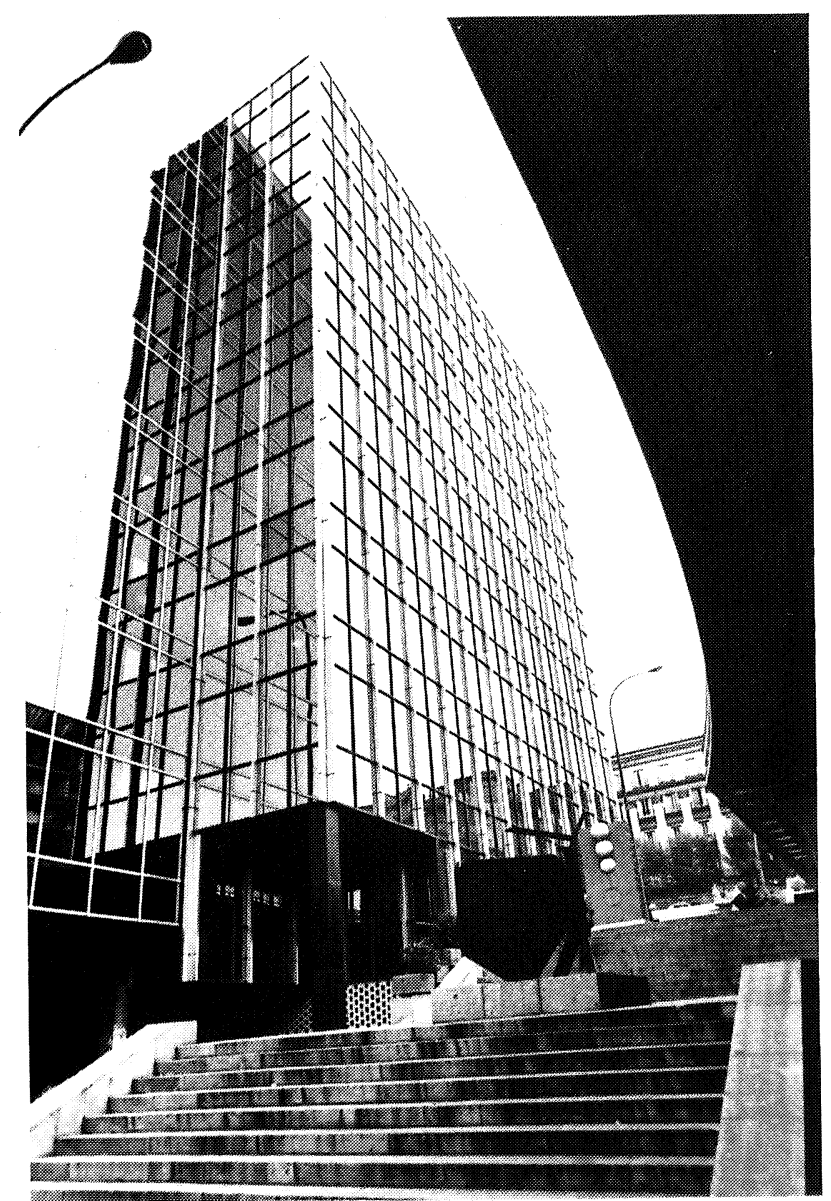

una fachada en muro-cortina con entramado muy pronunciado y tupido (Banco de París), a un muro-cortina completamente liso (Banco Peninsular), mediante una fachada en muro-cortina con un entramado estructural de dimensiones intermedias.

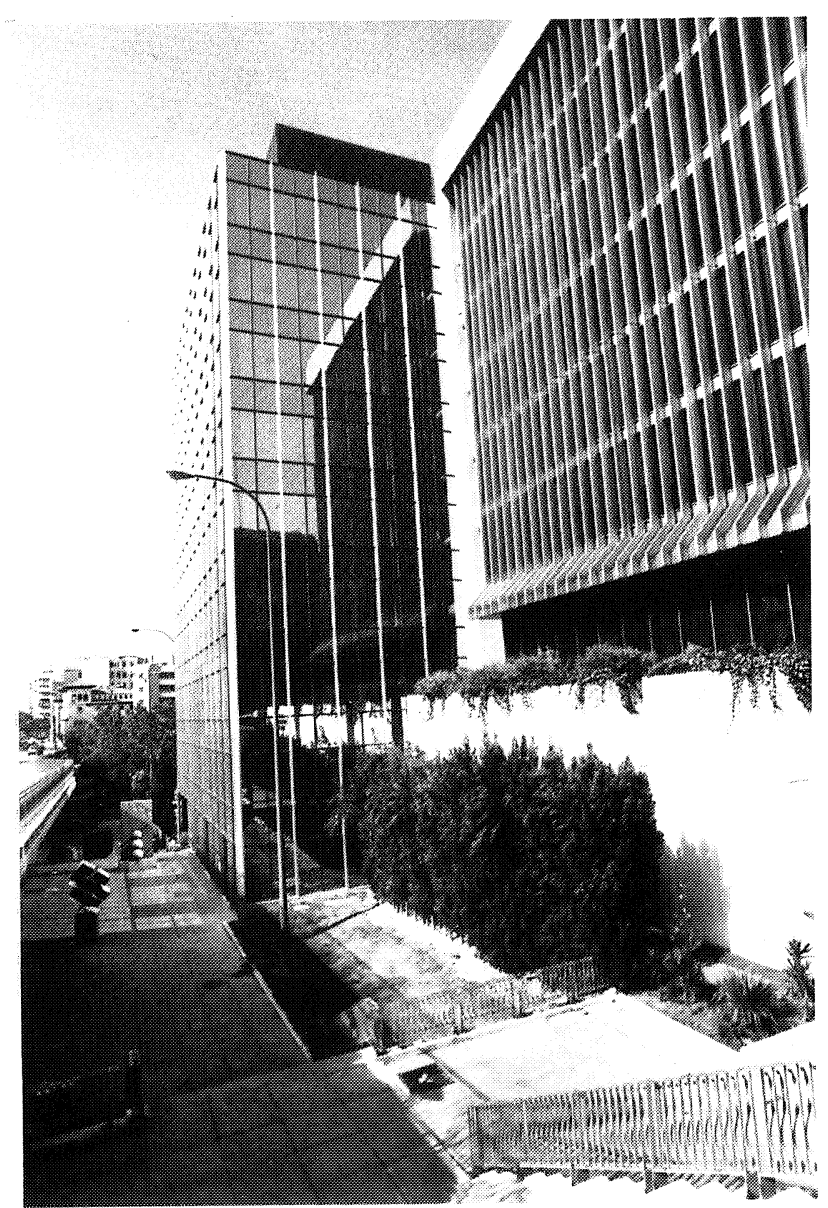



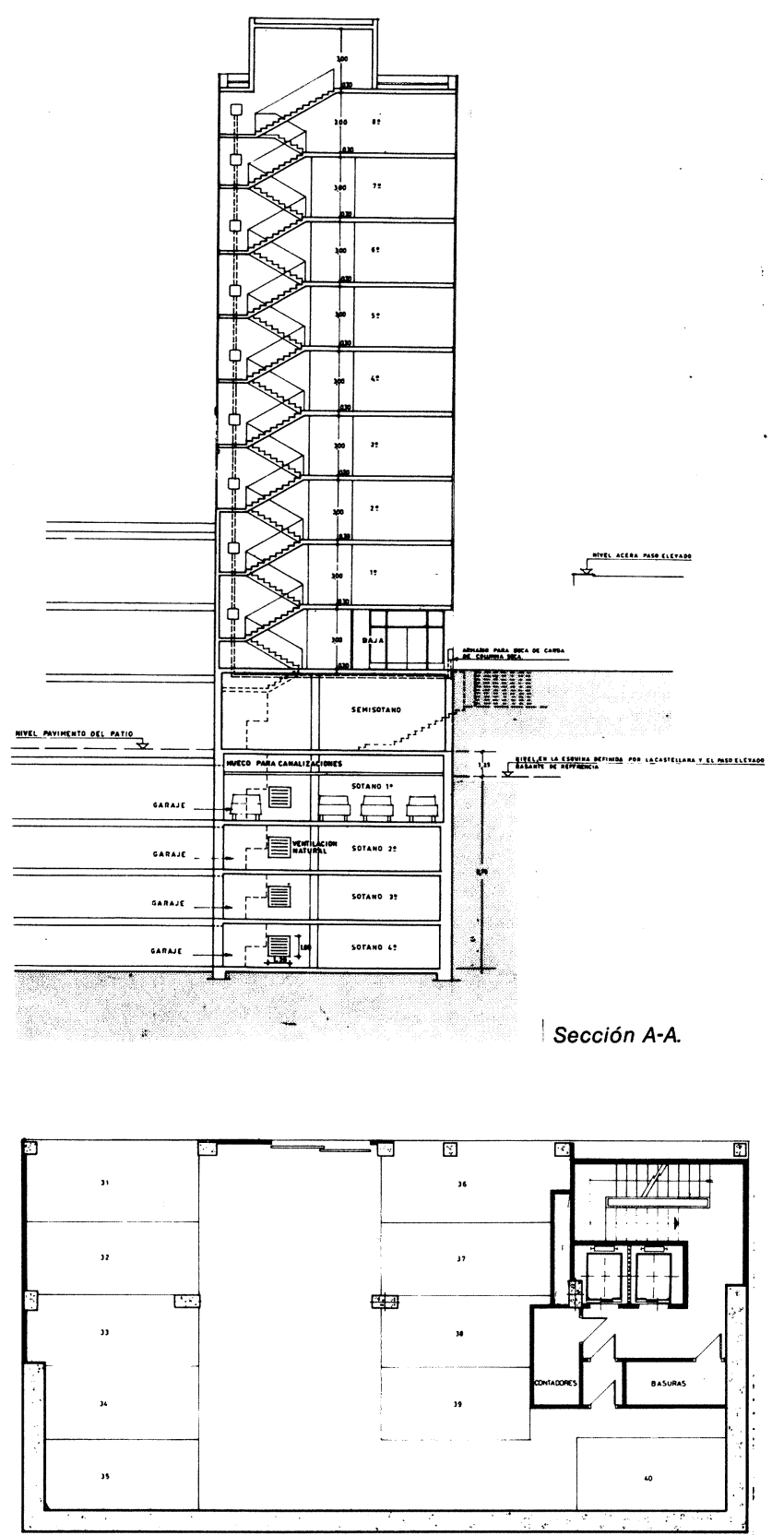

Sótano primero.

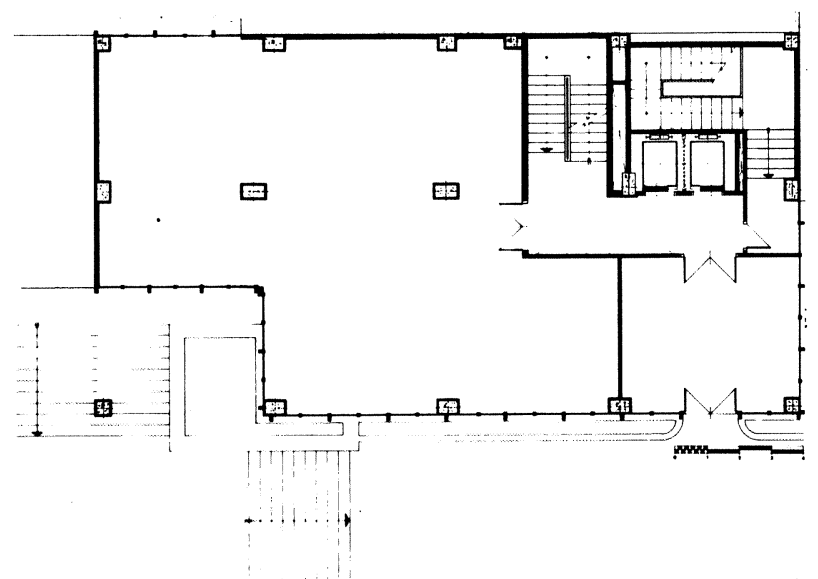

Planta tipo.
Se ha elegido el módulo de 1,75 m para la separación de los maineles verticales (elementos estructurales), ya que al ser 13 en fachada se pueden dividir los espacios interiores en 6 oficinas o despachos de 3,5 $\mathrm{m}$ de anchura, que es el tamaño ideal de despacho.

En las fachadas se combinan los elementos estructurales de aluminio con los paneles diáfanos de luna climalit logrando un ambiente armónico.

Para resaltar más la verticalidad del edificio, los maineles estructurales verticales se han construido con aluminio anodizado color oro, mientras que el resto del entramado de fachada con aluminio anodizado, en bronce, se confunde con el color de la luna climalit.

La cimentación es de muro pantalla perimetral de hormigón armado y zapatas de hormigón armado en los pilares centrales.

La estructura es de hormigón armado con forjados bidireccionales.

El pavimento del portal es de mármol blanco portugués; el de las escaleras, hall de ascensores y servicios, de mármol emperador.

El pavimento de las oficinas es de moqueta de color beige y el del garaje de slurry.

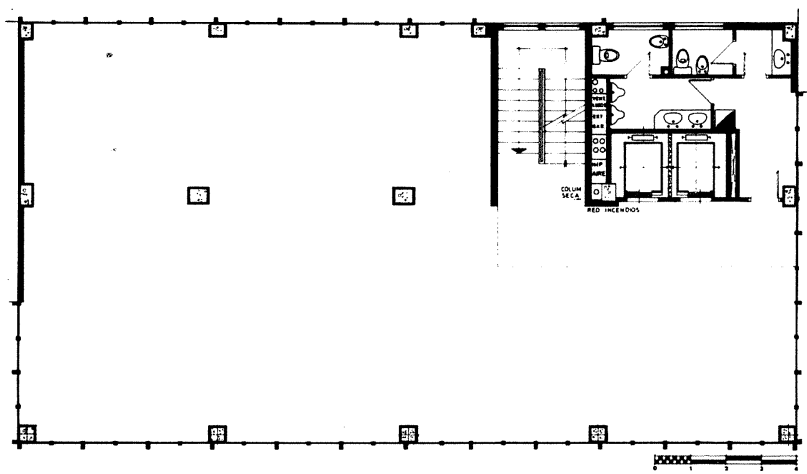

Planta baja.

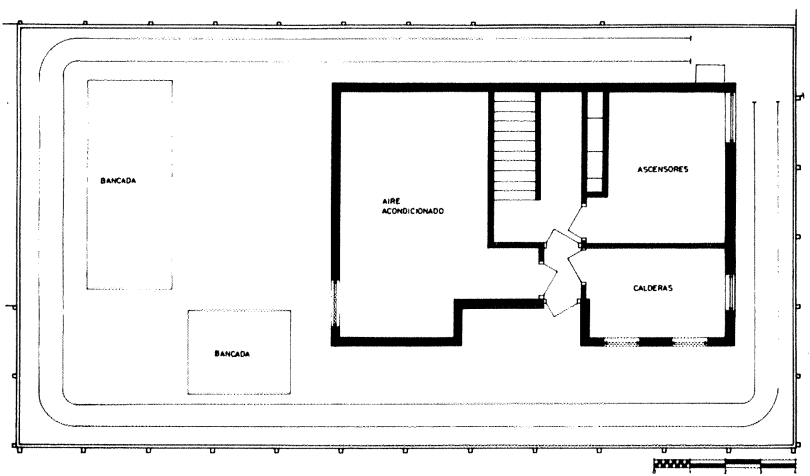

Planta de terraza. 


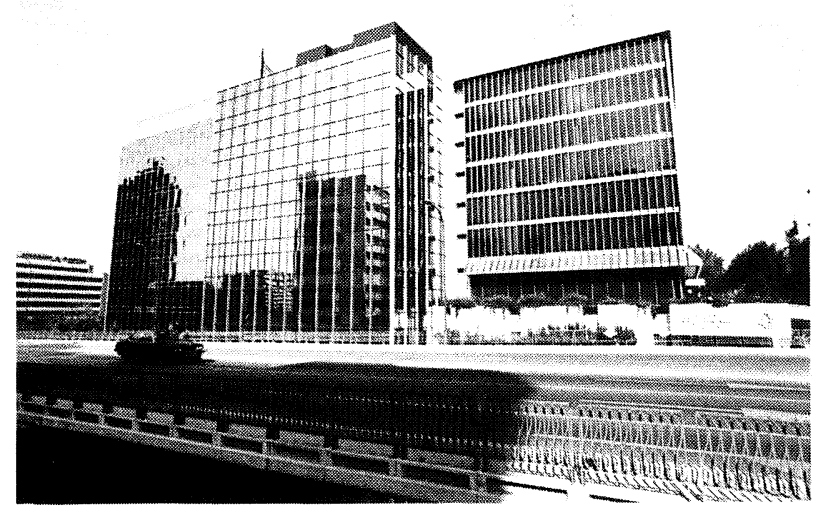

Las paredes del vestíbulo principal de entrada están revestidas de un empanelado mixto de madera lacada y espejos, con el fin de dar sensación de amplitud.

La barandilla de la escalera es de madera de roble sobre armadura de hierro combinada con baldosa de vidrio.

Los ascensores son de tipo Giesa de cabina con doble puerta y $1 \mathrm{~m} / \mathrm{s}$ de velocidad. Maniobra con memoria y

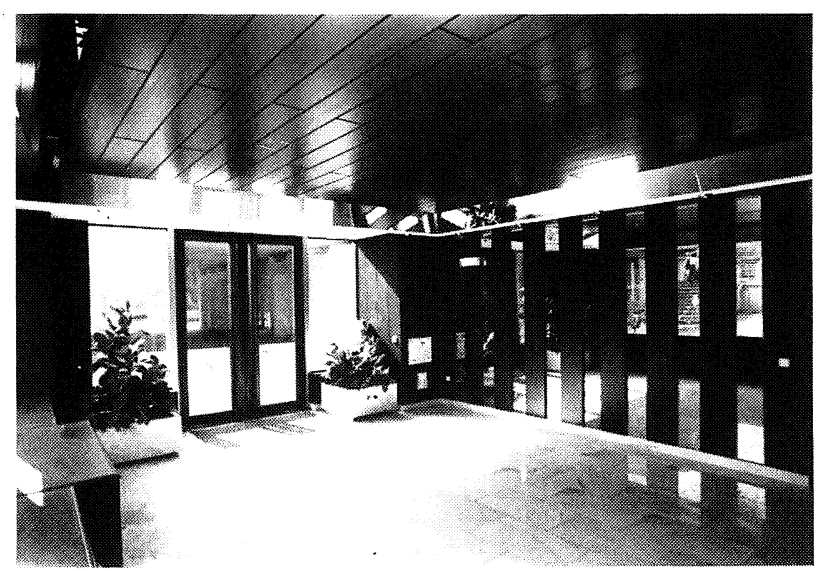

dos velocidades, micronivelación, paracaídas, puertas automáticas e indicador luminoso de posición.

La carpintería interior en madera de roble barnizada.

La calefacción y refrigeración interior se consigue mediante un sistema de aire acondicionado fancoil con consolas ocultas en los falsos techos.

A las cuatro plantas de garaje se accede a través del garaje común de la manzana.

$$
\text { 象农斿 }
$$

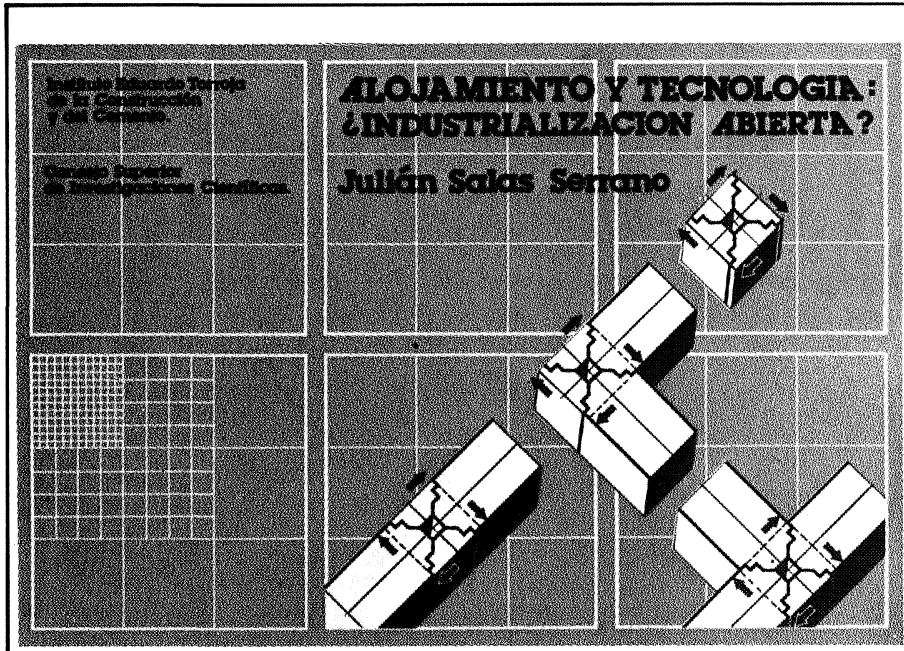

\section{ALOJAMIENTO Y TECNOLOGIA: ¿INDUSTRIALIZACION ABIERTA?}

JULIAN SALAS, ING. IND. (I.E.T.c.c.)

Un volumen de 160 páginas, 109 figuras y 16 tablas. Tamaño $240 \times 168 \mathrm{~mm}$. Encuadernado en rústica. Precios: España, 1.200 ptas; extranjero, 17 \$ USA.

\section{SUMARIO:}

Prólogo Prof. G. Ciribini.

\section{Introducción}

Capítulo 1.- La industrialización en las proclamas y manifiestos de arquitectura.

Capítulo 2.-¿Réquiem por la construcción industrializada?

Capítulo 3. - Algunos conceptos básicos.

Capítulo 4.-¿Proyecto tradicional, construcción industrializada?

Capítulo 5.-Componentes.

Capítulo 6. - La coordinación dimensional hoy.

Capítulo 7.-Flexibilidad, intercambiabilidad y catálogos. Capítulo 8. - Industrialización, normativa y calidad.

Capítulo 9.-Reflexiones finales.

publicación del

INSTITUTO EDUARDO TORROJA 